\title{
Upregulation of Polysialylated Neural Cell Adhesion Molecule in the Dorsal Hippocampus after Contextual Fear Conditioning Is Involved in Long-Term Memory Formation
}

\author{
Miguel A. Lopez-Fernandez, ${ }^{1}$ Marie-Françoise Montaron, ${ }^{2}$ Emilio Varea, ${ }^{1}$ Genevieve Rougon, ${ }^{4}$ Cesar Venero, ${ }^{3}$ \\ Djoher Nora Abrous, ${ }^{2}$ and Carmen Sandi ${ }^{1}$ \\ ${ }^{1}$ Brain Mind Institute, Swiss Federal Institute of Technology, CH-1015 Lausanne, Switzerland, ${ }^{2}$ Neurogenesis and Pathophysiology Laboratory, Bordeaux \\ Neuroscience Research Center, Institut National de la Santé et de la Recherche Médicale 862 and University of Bordeaux 2, Bordeaux Cedex 33077 , France, \\ ${ }^{3}$ Psychobiology Department, Universidad Nacional de Educacion a Distancia, 28040 Madrid, Spain, and ${ }^{4}$ Centre National de la Recherche Scientifique Unité \\ Mixte de Recherche 6216, Université de la Méditerranée, Institut de Biologie du Développement de Marseille-Luminy Case 907, Marseille Cedex 13288, \\ France
}

The role of the hippocampus in pavlovian fear conditioning is controversial. Although lesion and pharmacological inactivation studies have suggested a key role for the dorsal hippocampus in contextual fear conditioning, the involvement of the ventral part is still uncertain. Likewise, the debate is open with regard to the putative implication of each hippocampal subdivision in fear conditioning to a discrete conditioned stimulus. We explored the potential existence of dissociations occurring in the dorsal versus ventral hippocampus at the cellular level while dealing with either contextual or cued fear conditioning and focused in a molecular "signature" linked to structural plasticity, the polysialylated form of the neural cell adhesion molecule (PSA-NCAM). We found an upregulation of PSA-NCAM expression in the dorsal (but not ventral) dentate gyrus at $24 \mathrm{~h}$ after contextual (but not tone) fear conditioning. Specific removal of PSA through microinfusion of the enzyme endoneuraminidase- $\mathrm{N}$ in the dorsal (but not ventral) hippocampus reduced freezing responses to the conditioned context. Therefore, we present evidence for a specific role of PSA-NCAM in the dorsal hippocampus in the plasticity processes occurring during consolidation of the context representation after "standard" contextual fear conditioning. Interestingly, we also found that exposing animals just to the context induced an activation of PSA-NCAM in both dorsal and ventral dentate gyrus. Altogether, these findings highlighting the distinctive occurrence of these neuroplastic processes in the dorsal hippocampus during the standard contextual fear-conditioning task enlighten the ongoing debate about the involvement of these hippocampal subdivisions in pavlovian fear conditioning.

Key words: PSA-NCAM; hippocampus; fear conditioning; neurogenesis; learning and memory; rat

\section{Introduction}

Classical or pavlovian fear conditioning is a form of associative learning that presents attractive features to investigate the neural mechanisms of memory formation. In rodents, fearconditioning studies classically consist of the pairing of a conditioned stimulus (CS) with an aversive unconditioned stimulus (US; generally footshock), which induces conditioned fear responses (generally freezing). Different neural mechanisms seem to be involved depending on whether the CS is a relatively simple stimulus, such as a tone (unimodal), or the context (multimodal)

\footnotetext{
Received Dec. 2, 2006; revised March 17, 2007; accepted March 17, 2007.

This work was supported by grants from the European Union Fifth Framework Programme (FP) (QLRT-02187) and Sixth FP (FP6-2003-LIFESCIHEALTH-II-512012; PROMEMORIA), the Swiss National Science Foundation (3100A0108102), the Spanish Ministry of Science and Technology (MCYT) (BFI2003-07524 and BFU2006-01050), and Institut National de la Santé et de la Recherche Médicale. M.A.L.-F. was the recipient of a predoctoral fellowship from the $M C Y T$, and C.V. was the recipient of a contract from the Ramón y Cajal Program of the MCYT.

Correspondence should be addressed to Carmen Sandi, Laboratory of Behavioral Genetics, Brain and Mind Institute, Swiss Federal Institute of Technology, CH-1015 Lausanne, Switzerland. E-mail: carmen.sandi@epfl.ch.

DOI:10.1523/JNEUROSCI.0396-07.2007

Copyright $\odot 2007$ Society for Neuroscience $\quad$ 0270-6474/07/274552-10\$15.00/0
}

in which the US is delivered. Lesion experiments suggested that the amygdala is necessary for both types of conditioning, whereas the hippocampus is only required for contextual conditioning (Selden et al., 1991; Kim and Fanselow, 1992; Phillips and LeDoux, 1992).

However, the role of the hippocampus in fear conditioning is still a matter of debate. First, although there is general consensus that the hippocampus is not critically involved in the acquisition of cued fear conditioning (Phillips and LeDoux, 1992; Maren et al., 1997; Anagnostaras et al., 2001), some lesion (Richmond et al., 1999; Maren and Holt, 2004) and pharmacological interference (Bast et al., 2001) studies suggest a potential role. Second, whereas the involvement of the dorsal part of the hippocampus in contextual fear conditioning (CFC) is widely recognized (Sutherland and Rudy, 1989; Phillips and LeDoux, 1992, 1994; Young et al., 1994; Maren et al., 1997; O'Reilly and Rudy, 2001; Rudy et al., 2002), the involvement of the ventral part remains highly controversial (Anagnostaras et al., 2001; Bast et al., 2001; Kjelstrup et al., 2002; Bannerman et al., 2004; Rudy et al., 2004).

Our study aimed to provide insights into the potential re- 
gional (dorsal vs ventral) dissociations occurring in the hippocampus [with a particular focus in the dentate gyrus, which has been shown to be critical for the formation and retrieval of contextual fear memories (Lee and Kesner, 2004)] at the cellular level while dealing with either cued or contextual fear conditioning. For this purpose, we selected a molecular "signature" linked to hippocampal structural plasticity, the polysialylated form of the neural cell adhesion molecule (PSA-NCAM) (Bonfanti, 2006). PSA posttranslationally added to NCAM regulates NCAM interactions (Weinhold et al., 2005), decreasing cell adhesion and promoting structural remodeling (Rutishauser and Landmesser, 1996; Kiss and Rougon, 1997; Bonfanti, 2006). In addition to its functions on brain development, PSA-NCAM has been implicated in activity-dependent synaptic plasticity and memory function in adulthood (Murphy and Regan, 1998; Kiss and Muller, 2001; Dityatev and Schachner, 2003). A role for PSA-NCAM in fear conditioning has been suggested from studies on genetically modified mice (Angata et al., 2004; Senkov et al., 2006); nevertheless, permanent defects occurring in such models might obscure the dynamic function exerted by PSA-NCAM under normal conditions. Here, we asked (1) whether PSA-NCAM would be differentially modulated in the dorsal and ventral hippocampal dentate gyrus (DG) after either contextual or tone fearconditioning protocols; and (2) whether a loss of function of PSA obtained by enzymatic cleavage would affect the formation of fear memories for each fear learning modality.

\section{Materials and Methods \\ Animals}

Male Wistar rats [Complutense School of Medicine (Madrid, Spain) and Charles River Laboratories (Lyon, France)] weighing $\sim 250-350 \mathrm{~g}$ at the beginning of the experiments were housed in groups of three per cage, under light (12 h light/dark cycle; lights on at 7:00 A.M.) and temperature $\left(22 \pm 2^{\circ} \mathrm{C}\right)$ controlled conditions. Food and water were available ad libitum. All animals were handled for $2 \mathrm{~min} / \mathrm{d}$ during the $3 \mathrm{~d}$ preceding the first behavioral test. Animal care procedures were conducted in accordance with the guidelines set by the European Community Council Directives (86/609/EEC) and the Cantonal Veterinary Authorities (Vaud, Switzerland).

\section{Fear conditioning}

Training and testing took place in a rodent observation cage $(30 \times 37 \times$ $25 \mathrm{~cm}$ ) that was placed in a sound-attenuating chamber. The side walls of the observation cage were constructed of stainless steel, and the door was constructed of Plexiglas. The floor consisted of 20 steel rods through which a scrambled shock from a shock generator (Panlab, Barcelona, Spain) could be delivered. Each observation cage was cleaned with a $0.1 \%$ acetic acid solution before and after each session. Ventilation fans provided a background noise of $68 \mathrm{~dB}$, and a $20 \mathrm{~W}$ white lightbulb illuminated the chamber.

On the conditioning/training day, each rat was transported from the colony room to the behavioral laboratory (situated in adjacent rooms) and placed in the conditioning chamber. In the CFC version, after $3 \mathrm{~min}$ of exposure to the cage, rats received three $1 \mathrm{~s}$ footshocks $(0.4$ or $1 \mathrm{~mA}$ intensity) with an intertrial interval of $60 \mathrm{~s}$. In the auditory-cued (tone) fear-conditioning (TFC) version, the same protocol of shock delivery was followed, with the exception that a $20 \mathrm{~s}$ presentation of a tone $(85 \mathrm{~dB}$ sound at $1000 \mathrm{~Hz}$ ) preceded each footshock. Rats were removed from the conditioning chamber $30 \mathrm{~s}$ after the final shock presentation and returned to their home cages. Therefore, the conditioning session lasted 5.5 $\min$.

Testing was performed at different times [i.e., $30 \mathrm{~min}$ and $24 \mathrm{~h}$ after training in experiments involving PSA-NCAM quantification by immunohistochemistry and $48 \mathrm{~h}$ after training in experiments involving enzymatic cleavage of PSA with endoneuraminidase-N (endo-N)]. In the CFC version, rats were placed back into the original training context for $8 \mathrm{~min}$, during which no footshock was delivered. In the TFC version, animals were placed into a novel context (same cages, but with different walls, floor, and background odor), and, after a $3 \mathrm{~min}$ baseline period, they were continuously reexposed to the tone (same characteristics as at conditioning) for $5 \mathrm{~min}$, but in the absence of shocks.

In addition, two experiments were performed to study the separate impact of "shock" and "context" on PSA-NCAM expression in the DG. To evaluate the effect of the shock and minimize context exposure ("immediate shock" condition), rats were given one $2 \mathrm{~s}$ footshock (1 mA) immediately after being introduced in the novel context and quickly removed and returned to their home cage. To assess for the impact of the context ("context exposure" condition), rats were allowed to explore the novel context for $5.5 \mathrm{~min}$ without receiving any additional stimulation.

The animals' behavior was video recorded and later scored by an observer blind to the treatment condition. Using a time-sampling procedure every $2 \mathrm{~s}$, each rat was scored blindly as either freezing or active at the instant the sample was taken. Freezing was defined as behavioral immobility except for movement needed for respiration. All experiments were conducted between 8:30 A.M. and 14:00 P.M. to avoid the influence of circadian hormonal fluctuations.

\section{Surgery}

Experiments to study the functional implications of PSA-NCAM by enzymatic removal involved surgery and cannulation. At least 1 week after arrival, rats subjected to pharmacological experiments were implanted with stainless steel guide cannulas aimed at the dorsal or ventral hippocampus. They were anesthetized with an isoflurane vaporizer (VIP 3000; MDS Matrix, Bern, Switzerland) and mounted in a stereotaxic apparatus. The scalp was incised and retracted, and the head was positioned to place bregma and lambda in the same horizontal plane. Small holes were drilled through the skull for bilateral placement of a stainless double 22 gauge Teflon guide cannula (Plastic One, Roanoke, VA) fitted with a removable dummy cannula, above either the dorsal $(3.8 \mathrm{~mm}$ posterior, $2.5 \mathrm{~mm}$ lateral, and $2.4 \mathrm{~mm}$ deep relative to bregma) or the ventral ( $5.20 \mathrm{~mm}$ posterior, $4.5 \mathrm{~mm}$ lateral, and $6 \mathrm{~mm}$ deep) hippocampus. The coordinates were based on the atlas of Paxinos and Watson (1986). The cannula was fixed to the skull with two anchoring screws and dental acrylic (Duralay 2244; Reliance, Worth, IL). Once operated, animals were housed individually and left to recover for at least $5 \mathrm{~d}$ before any behavioral test.

\section{Endo-N injection procedures}

To eliminate PSA-NCAM, the highly specific PSA-glycosidase termed endo- $\mathrm{N}$ was used. The dummy cannulas were removed from the guide cannulas and replaced with injection cannulas (Plastic One) attached to $100 \mu \mathrm{l}$ Hamilton syringes via polyurethane tubing (fluorinated ethylene propylene; $0.12 \mathrm{~mm}$ diameter CMA; Microdialysis AB, Stockholm, Sweden). The syringes were mounted onto an infusion pump (Harvard Apparatus, Cambridge, MA). A flexible swivel attached to the rat allowed the enzyme to be administered while the animal was conscious and moving ad libitum in a cage. For endo-N or vehicle infusions, injectors were inserted so that they extended $1 \mathrm{~mm}$ below the guide cannulas. One day before training in contextual or auditory-cued fear-conditioning, rats were injected with either endo-N (0.7 U/hemisphere; Abcys, Paris, France) or vehicle [artificial CSF (aCSF)] at $0.5 \mu \mathrm{l} / \mathrm{min}$ for $2 \mathrm{~min}$. The injection cannulas were left in place for an additional $2 \mathrm{~min}$ to prevent back-leakage of the solutions. Moreover, an additional experiment was performed involving either vehicle or endo- $\mathrm{N}$ injections immediately after training rats in the contextual fear-conditioning version. Finally, to test for the specificity of the effects observed with endo- $\mathrm{N}$ injections, an additional experiment involved the administration of heat-inactivated endo- $\mathrm{N}$ ( $0.7 \mathrm{U} /$ hemisphere; same infusion conditions as above) into the dorsal hippocampus. This procedure is frequently and successfully used in the literature to account for the specificity of endo-N effects (Rutishauser et al., 1988; Daston et al., 1996; Lenka et al., 2002; Burgess and Aubert, 2006).

\section{Control for cannula placement and cannula diffusion}

At the end of all infusion experiments, rats were anesthetized with an overdose of pentobarbital $(120 \mathrm{mg} / \mathrm{kg})$. Then, a cresyl violet solution was administered in the same way as the endo- $\mathrm{N}$ and the control solutions to 
verify the cannula placement and endo-N efficacy. After decapitation, the brains were removed and examined for the presence of cresyl violet in the hippocampus. Only the rats with correct cannula implantation were included in the analysis. In a previous immunohistochemical study, we observed that, under our experimental conditions, endo- $\mathrm{N}$ diffusion was still evident $96 \mathrm{~h}$ after injection, showing an effectiveness of $\sim 90 \%$. Only one of eight injected brains showed an inaccurate diffusion.

\section{BrdU injections}

Rats of the first experiment were treated 1 week before exposure to either contextual or tone fear conditioning with 5-bromo-2-deoxyuridine (BrdU; $200 \mathrm{mg} / \mathrm{kg}$, i.p.) dissolved in a phosphate buffer (0.1 M, pH 8.4), following a procedure published previously (Gould et al., 1999).

\section{Immunohistochemistry}

In experiments designed to evaluate the modulation of hippocampal PSA-NCAM by fear conditioning, rats were perfused immediately after the testing session. In the experiment designed to evaluate the impact of the testing session, a group of animals ("only testing" condition) were perfused $24 \mathrm{~h}$ after training in the fear-conditioning task. In the experiments evaluating the impact of either the shock or the context separately, the animals were also perfused $24 \mathrm{~h}$ after exposure to these stimuli. For perfusion, rats were first deeply anesthetized $(120 \mathrm{mg} / \mathrm{kg}$ pentobarbital $)$ and then transcardially perfused with $200 \mathrm{ml}$ of PBS, pH 7.3, containing heparin $\left(5 \times 10^{4} \mathrm{U} / \mathrm{ml}\right)$, followed by $300 \mathrm{ml}$ of $4 \%$ paraformaldehyde in $0.1 \mathrm{~m}$ phosphate buffer, $\mathrm{pH}$ 7.3. Brains were postfixed at least for $48 \mathrm{~h}$ in picric acid-formaldehyde. Then, $50 \mu \mathrm{m}$ frontal sections were cut on a vibratome (VT 1000S; Leica, Glattbrugg, Switzerland). Free-floating sections were processed according to a standard immunohistochemical procedure (Lemaire et al., 2006). One in 10 sections was treated for PSANCAM immunoreactivity (PSA-NCAM-IR) using a mouse anti-Men B monoclonal antibody [1:4000 (Rougon et al., 1986)]. For BrdU labeling, adjacent sections were treated with $2 \mathrm{~N} \mathrm{HCl}\left(30 \mathrm{~min}\right.$ at $\left.37^{\circ} \mathrm{C}\right)$ and then rinsed in borate buffer during $5 \mathrm{~min}(0.1 \mathrm{M} ; \mathrm{pH}$ 8.4). They were incubated with a mouse monoclonal anti-BrdU antibody (1:200; Dako, High Wycombe, UK). Sections were processed in parallel, and immunoreactivities were visualized by the biotin-streptavidin technique (ABC kit; Dako) using 3,3'-diaminobenzidine as chromogen.

\section{Stereological analysis}

The total number of PSA-NCAM-IR cells in the subgranular and granule cell layers (gcls) of the DG was estimated using a modified version of the optical fractionator method on a systematic random sampling of every tenth section along the rostrocaudal axis of the hippocampal formation (HF) (Lemaire et al., 2006). Cell quantification was performed in the left hemisphere, apart from one experiment (see description in Results) in which the evaluation was done in both hemispheres.

\section{Histological characterization of the effectiveness of endo-N injections}

Four days after injection of endo-N, PSA immunoreactivity was evaluated in eight animals, using $50 \mu \mathrm{m}$ coronal sections of the brain of adult rat, covering the rostrocaudal extension of the hippocampal formation, from the very rostral edge of the hippocampus to $\sim 4-5 \mathrm{~mm}$ caudally. Enzyme diffusion in the dorsal DG, CA1, and CA3 regions were manually traced, and the percentage of the area lacking PSA immunoreactivity in the dorsal hippocampus was quantified using image analysis software (Leica QWin).

\section{Behavioral characterization of locomotion and unconditioned fear} In an experiment, we evaluated the potential effects of dorsal hippocampal endo-N infusions in behavioral responses (such as locomotion/exploration, unconditioned fear, or stress reactivity) that could confound its effectiveness in the fear-conditioning paradigms. Vehicle- and endo$\mathrm{N}$-infused animals were, thus, tested for their behavior in the open field and startle response at 48 and $72 \mathrm{~h}$, respectively, after injections.

Open field. Emotional responses and locomotor reactivity of rats to a novel environment were assessed in the open field test, which consists of placing the animals in a circular arena ( $1.50 \mathrm{~m}$ diameter; $32 \mathrm{~cm}$ height). For analysis, the floor was divided into two virtual concentric parts, with the inner zone considered as the middle of the arena ( $1 \mathrm{~m}$ diameter) and an outer zone made up of the remaining area along the sidewalls. The animals were placed in the center of the arena at the start of the test, and their behavior was monitored for $10 \mathrm{~min}$ using a video camera mounted on the ceiling above the center of the arena. A computerized tracking system (Ethovision 3.1.16; Noldus IT, Wageningen, The Netherlands) recorded the amount of time spent in each zone, the total locomotion, and the number of times they crossed between inner and outer zones. The behavioral analysis included the following attributes: (1) time spent rearing; (2) time spent grooming; (3) time spent frozen; and (4) number of defecations. All of these variables were studied to investigate whether endo-N microinjections affect locomotor activity and anxiety behavior.

Startle response. Acoustic startle response was measured in an SR-Lab Startle Reflex System (San Diego Instruments, San Diego, CA). Within the system's sound-proof chamber, rats were placed in a Plexiglas cylinder $(9 \mathrm{~cm}$ diameter), large enough to hold the animals with minimal restraint enclosure, that was fixed on a motion-sensitive platform. The platform sits on a base, under which is a piezoelectric accelerometer, which detects whole-body startle responses. Signals from the accelerometer were rectified, digitized, and stored on computer by the SR-Lab program (San Diego Instruments). Chambers were calibrated each day and matched for sound intensity. The white background noise was adjusted to a constant $70 \mathrm{~dB}$. After a 5 min acclimatization period, two blocks of 10 randomly startle-only trials $(115 \mathrm{~dB} ; 40 \mathrm{~ms})$ were presented.

\section{Statistical analysis}

The behavioral data regarding the time each rat spent freezing was transformed to a percentage freezing per minute in each session and then, for statistical tests and figure representation, percentage freezing per minute was averaged for all minutes in each session belonging to the same category (i.e., preshock or postshock freezing during the conditioning sessions; and freezing to the 8 min context test or freezing to the 5 min tone test during the respective testing sessions). All results were expressed as mean \pm SEM and analyzed by ANOVA or Student's $t$ test, as appropriate. Differences in PSA-NCAM-IR between different training and pharmacological conditions were further evaluated for significance with post hoc Tukey's or Student's $t$ tests when appropriate. In all cases, significance of results was accepted at $p \leq 0.05$.

\section{Results}

\section{Contextual, but not tone, fear conditioning increases PSA-} NCAM expression in the DG $24 \mathrm{~h}$ after training

Animals were trained in either contextual or tone fearconditioning protocols (at 0.4 or $1 \mathrm{~mA}$ ) and then tested for their respective modality-specific fear memories at either $30 \mathrm{~min}$ or $24 \mathrm{~h}$ later $(n=12$ rats per group for each shock intensity, testing session, and conditioning modality, as well as for an additional naive control group). Animals trained at $1 \mathrm{~mA}$, in either the contextual (Fig. $1 A$ ) or the tone (Fig. $1 B$ ) fear-conditioning protocol, always showed higher freezing levels than those trained at $0.4 \mathrm{~mA}$, both during the postshock period at training (all $p<$ 0.05 ) and at any of the testing times (all $p<0.05$ ).

Immediately after the testing session ( $30 \mathrm{~min}$ or $24 \mathrm{~h}$ ), animals were perfused for the anatomical study. Immunohistochemistry revealed a discrete group of PSA-NCAM neurons that were localized in the innermost portion of the gcl at the interface of the hilus (Figs. 1C,D). These cells displayed the characteristic morphology of granule neurons, with their dendrites extending through the granule cell layer into the molecular layer and their axons, the mossy fibers, projecting onto the CA3 subfield of the hippocampus. As shown in Figure $1 E$, there was an effect of CFC in the number of PSA-NCAM-IR cells $\left(F_{(4,46)}=4.767 ; p=0.003\right)$. Compared with controls, animals trained in the CFC modality showed an increased number of PSA-NCAM-IR cells in the DG $24 \mathrm{~h}\left(F_{(2,25)}=5.136 ; p=0.014\right)$ but not $30 \mathrm{~min}\left[F_{(2,32)}=0.258\right.$; not significant (n.s.)] after training. The increase observed at $24 \mathrm{~h}$, particularly prominent in the $1 \mathrm{~mA}$-trained group, was 


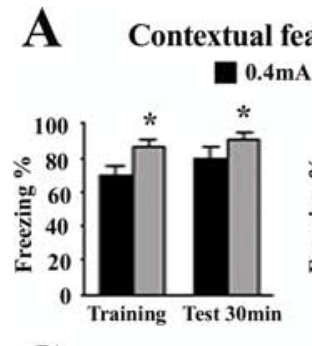

C

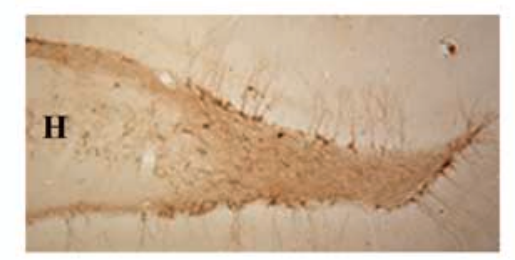

$\mathbf{E}$

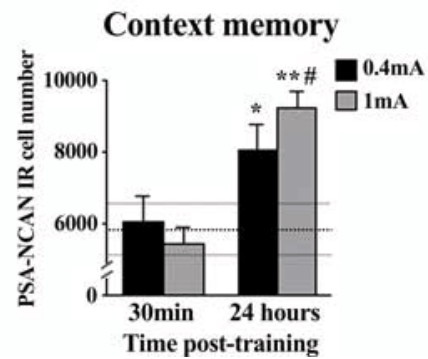

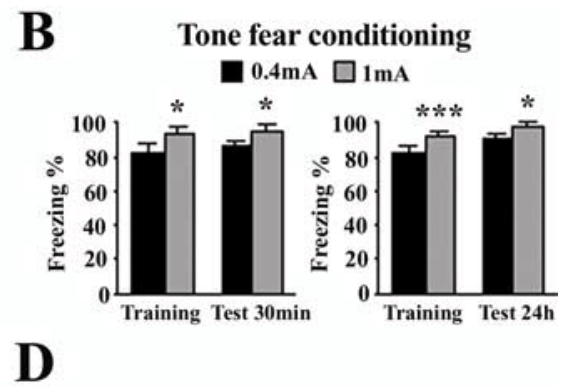

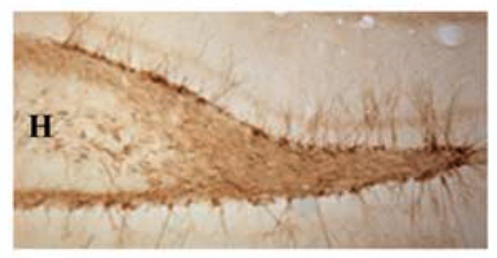

F

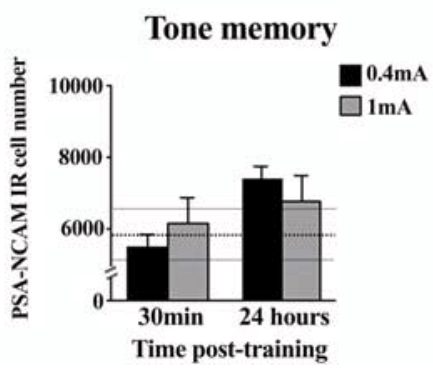

Figure 1. Contextual, but not tone, fear conditioning increases PSA-NCAM expression in the dentate gyrus of the hippocampus. $\boldsymbol{A}, \boldsymbol{B}$, Percentage of time spent freezing during the context $(\boldsymbol{A})$ and tone $(\boldsymbol{B})$ tests, $30 \mathrm{~min}$ or $24 \mathrm{~h}$ after fear conditioning under two different shock intensities ( 0.4 or $1 \mathrm{~mA}$ ). C, D, PSA-NCAM-labeled cells in the dentate gyrus. Representative photomicrographs of PSA-NCAM staining of the dentate gyrus in a naive rat $(\boldsymbol{C})$ and in an animal submitted to contextual fear conditioning ( $1 \mathrm{~mA}$ shock intensity) (D). $\boldsymbol{E}, \boldsymbol{F}$, Number of polysialylated cells in the dentate gyrus $30 \mathrm{~min}$ or $24 \mathrm{~h}$ after training animals in either a contextual $(\boldsymbol{E})$ or a tone $(\boldsymbol{F})$ fear-conditioning paradigm. Data represent mean \pm SEM. Thickest horizontal discontinuous line represents mean values of the naive group, and thinner ones represent SEM values of this group. H, Hilus. ${ }^{*} p<0.05$, ${ }^{* *} p<0.01$, ${ }^{* *} p<$ 0.001 versus naive; ${ }^{\#} p<0.001$ versus $1 \mathrm{~mA}$ group in the 30 min test.

significant for either shock condition $(0.4 \mathrm{~mA}, p=0.05 ; 1 \mathrm{~mA}$, $p<0.005)$. However, training in the TFC modality, at any of the shock conditions, failed to induce changes in DG PSA-NCAM expression as evaluated at both $30 \mathrm{~min}$ and $24 \mathrm{~h}$ after training $\left(F_{(4,46)}=1.823\right.$; n.s. $)($ Fig. $1 F)$.

It is important to note that freezing values to the conditioned context differ between animals trained in the CFC and TFC modalities. Substantial evidence from our laboratory indicates that whereas training rats in the CFC protocol at $1 \mathrm{~mA}$ leads to $\sim 90 \pm$ $10 \%$ (and $0.4 \mathrm{~mA}$ to $\sim 70 \pm 10 \%$ ) freezing during the context memory test, training in the auditory fear conditioning at $1 \mathrm{~mA}$ typically leads to much lower freezing values when animals are reexposed to the conditioning context [i.e., $\sim 25 \pm 5 \%$ (and $\sim 18 \pm 5 \%$ ) freezing (Cordero et al., 1998, 2002; Markram et al., 2007b)].

\section{Effects on the survival of recently generated cells}

Because PSA-NCAM has been associated with hippocampal neurogenesis, we verified whether the above increase in PSA-NCAM expression was associated with an increase in cell genesis. Animals were injected with BrdU 1 week before testing. This time schedule was chosen because (1) it has been shown that newborn cells in the adult DG express PSA-NCAM $\sim 1$ week after being born (Seki and Arai, 1993); and (2) it was previously shown that training rats in another pavlovian conditioning task prevented the survival of cells generated 1 week before testing (Gould et al., 1999). The newly born BrdU-labeled cells were isolated, round, and large and were residing either in the subgranular zone or in the gcl (data not shown). We found that the total number of BrdUlabeled cells was not influenced by training in the contextual or the auditory version of the fear conditioning, at any of the shock conditions (n.s.; data not shown).

\section{Immediate shock exposure failed to affect PSA-NCAM expression $24 \mathrm{~h}$ afterward, but context exposure increased PSA-NCAM levels both in dorsal and ventral DG}

Increased PSA-NCAM levels observed after CFC might be argued to be induced by the shock, the context exploration, or the conjunction of both (i.e., the latter related to experiencing the shock in a particular context and, therefore, putative to $\mathrm{CFC}$ ). Identifying the key element(s) involved in the observed effect is relevant because a rat shocked immediately after being introduced into a new context and removed rapidly afterward (immediate shock condition) does not develop fear for that context (Fanselow, 2000). However, if it was preexposed to the context on the previous day, the immediate shock results in significant contextual fear conditioning (Fanselow, 2000; Rudy and O'Reilly, 2001). This is termed "context preexposure facilitation effect" (CPFE) and has been interpreted as a lack of time in the immediate shock condition to encode a representation of the context before immediate shock. Thus, context preexposure facilitates the amount of conditioning produced by immediate shock through the association of the retrieved memory representation to the immediate shock (Rudy and O'Reilly, 2001; Rudy et al., 2002). This implies that any putative mechanism related to CFC should not be elicited by the immediate shock condition. It also suggests that mechanisms involved in CFC might be already triggered by the mere exposure to the context. To assess these possibilities, we performed two experiments in which PSA-NCAM levels in control animals were compared with those displayed by animals that either were exposed to shock with minimal context exposure (immediate shock condition) or were just allowed to explore the new context (context exposure condition).

As shown in Figure 2A, immediate shock delivery (one $2 \mathrm{~s}, 1$ $\mathrm{mA}$ footshock) with a minimal context exposure did not alter PSA-NCAM levels in the total DG $\left(t_{(8)}=0.80\right.$; n.s. $)$, the dorsal part $\left(t_{(8)}=0.371 ;\right.$ n.s. $)$, or the ventral part $\left(t_{(8)}=1.238\right.$; n.s. $)$, compared with controls. As to the impact of context exploration (without shock), context exposure resulted in increased levels of PSA-NCAM expression both in the dorsal $\left(t_{(14)}=3.680\right.$; $p=$ $0.002)$ and ventral $\left(t_{(14)}=3.715 ; p=0.002\right)$ parts of the DG, an effect which was also apparent on total DG expression $\left(t_{(14)}=\right.$ 3.981; $p=0.001)($ Fig. $2 B)$. 

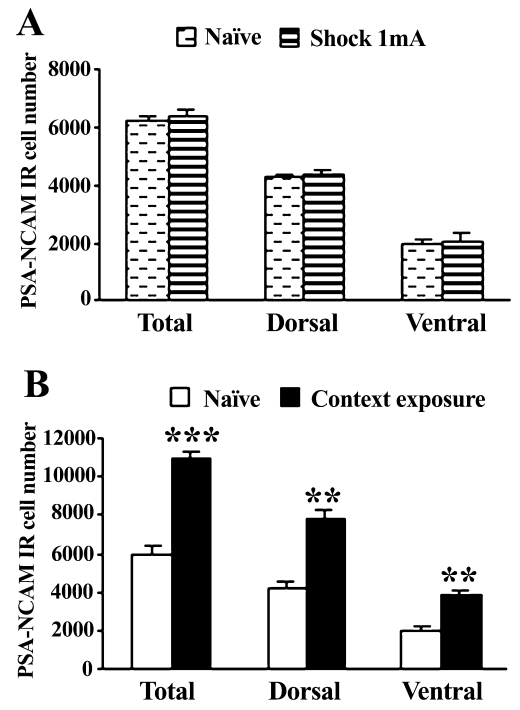

Figure 2. Immediate shock does not regulate PSA-NCAM levels in the dentate gyrus, but context exposure upregulates its expression both in the dorsal and ventral parts. $A, B$, Number of polysialylated cells in the dentate gyrus $24 \mathrm{~h}$ after submitting animals either to a footshock (2 $s ; 1 \mathrm{~mA})$ with minimal context exposure $(\boldsymbol{A})$ or just to the context $(\boldsymbol{B})$. Data represent mean \pm SEM. ${ }^{* *} p<0.01,{ }^{* * *} p<0.001$ versus naive.

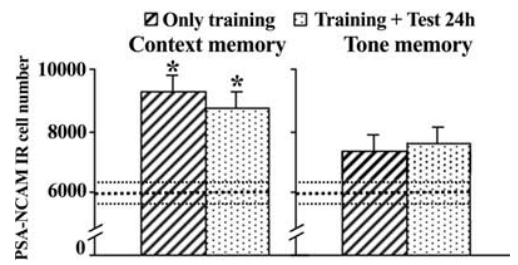

Figure 3. Training increases the number of PSA-NCAM-labeled cells in the dentate gyrus $24 \mathrm{~h}$ after contextual fear conditioning. Left, Quantitative analysis of polysialylated cell number in the $D G$ of two independent groups of animals, both of them trained in the contextual fear conditioning $(1 \mathrm{~mA})$, but one of them also tested $24 \mathrm{~h}$ later (Training + Test $24 \mathrm{~h}$ ) and the other left undisturbed after training (Only training). Both groups of animals were perfused $24 \mathrm{~h}$ after training. Right, The same experimental protocol was followed to check independently the effects of training and retrieval in the tone fear-conditioning protocol on the number of hippocampal PSA-NCAM-labeled cells. Data represent mean \pm SEM. Thickest horizontal discontinuous line represents mean values of the naive group, and thinner ones represent SEM values of this group. ${ }^{*} p<0.05$ versus naive.

The increase in PSA-NCAM expression observed $24 \mathrm{~h}$ after contextual fear conditioning is induced by training, not by the $24 \mathrm{~h}$ retrieval test

Because our first experiment evaluated PSA-NCAM expression in animals that were both submitted to fear conditioning and tested for their retrieval levels $24 \mathrm{~h}$ later (just before perfusion for immunohistochemistry), it was not possible to dissociate between the potential effects induced by each of these behavioral sessions. We therefore designed an experiment to specifically address this question, by including groups trained in the CFC (1 $\mathrm{mA}$ ) that were then either tested $24 \mathrm{~h}$ later followed by perfusion ("Test $24 \mathrm{~h}$ "; $n=11$ ) or left undisturbed from training until perfusion 24 h later ("Only training"; $n=12$ ). For comparison, we also followed the same experimental design in animals trained in the TFC protocol (1 mA) and included a control group consisting of naive animals $(n=12)$.

As shown in Figure 3 (left), CFC induced an increase in PSANCAM expression in the DG when evaluated $24 \mathrm{~h}$ after training $\left(F_{(2,26)}=3.621 ; p=0.041\right)$ that was independent of the retrieval session given just before perfusion, because this increase was ev- ident in both groups Test $24 \mathrm{~h}(p<0.05)$ and Only training $(p<$ $0.05)$ when compared with controls. This result indicates that PSA-NCAM increase observed $24 \mathrm{~h}$ after training is related to the contextual conditioning experience at training. In addition, we confirmed a lack of effect of TFC on dentate PSA-NCAM expression $\left(F_{(2,27)}=0.598\right.$; n.s. $)$, because none of the experimental groups, either just trained or trained and tested $24 \mathrm{~h}$ later, display significant changes (Fig. 3, right).

The dorsal, but not the ventral, hippocampal DG shows increased PSA-NCAM expression after contextual fear conditioning

We next addressed whether the observed increase of PSA-NCAM expression in the dorsal hippocampus after contextual training (1 $\mathrm{mA}$ ) is specifically circumscribed to a particular hippocampal subdivision (i.e., dorsal vs ventral). For comparison, we also evaluated the expression of PSA-NCAM in the dorsal and ventral dentate subdivisions in animals submitted to TFC ( $1 \mathrm{~mA})$. Such study was performed in the sections of the experiment just reported above (i.e., corresponding to results presented in Fig. 3). Moreover, because lateralization in the mechanisms involved in context-aversive memories has been described (Desmedt et al., 2003), we also assessed PSA-NCAM expression in the DG separately for each hemisphere. Because no interhemispheric differences were observed in any of the conditions examined (data not shown), for the sake of clarity and to allow comparison with other experiments, we present here the results corresponding to the left DG.

When analyzed $24 \mathrm{~h}$ after training, the increase in PSA-NCAM expression induced by CFC is observed at the level of the dorsal DG (Fig. 4A, left) $\left(F_{(2,26)}=8.802 ; p<0.001\right.$; Test $24 \mathrm{~h}, p<0.05$; Only training, $p<0.05$ vs controls), but no significant changes are apparent in the ventral part of the DG (Fig. $4 B$, left $)\left(F_{(2,26)}=\right.$ 1.339 ; n.s.). The increase in PSA-NCAM-IR in the dorsal DG was bilateral, with both trained groups showing higher expression than the naive group in the left and right subdivisions (data not shown; all $p<0.05$ ). Again, none of the DG subdivisions [dorsal (Fig. $4 A$, right $)\left(F_{(2,27)}=1.201\right.$; n.s.) or ventral (Fig. $4 B$, left) $\left(F_{(2,27)}=1.333\right.$; n.s. $\left.)\right]$ showed significant changes in animals trained in the TFC paradigm.

Enzymatic removal of PSA-NCAM in the dorsal hippocampus impairs long-term memory for contextual, but not tone, fear conditioning

Then, we performed a series of experiments to evaluate the effect of pharmacologically removing PSA-NCAM on each of the fearconditioning modalities, by injecting bilaterally the PSA-specific glycosidase endo- $\mathrm{N}$ in either of the hippocampal subdivisions (Fig. 5) ( $n=5-9$ per group). Infusions of endo- $\mathrm{N}$ given into the dorsal hippocampus $24 \mathrm{~h}$ before training did not affect immediate freezing responses whatever the shock intensities delivered ( 0.4 or $1 \mathrm{~mA}$ ) during conditioning in either the contextual (all n.s.) (Fig. $5 A$ ) or the tone (all n.s.) (Fig. $5 B$ ) modality. When retention was tested $48 \mathrm{~h}$ after conditioning, although no effect of the treatment was observed for TFC at any of the shock intensities $\left[0.4 \mathrm{~mA}, t_{(15)}=1.141\right.$; n.s. (Fig. $5 C$ ) $; 1 \mathrm{~mA}, t_{(16)}=0.742$; n.s. (Fig. $5 D)$ ], endo- $\mathrm{N}$ injections significantly reduced freezing responses in the CFC paradigm at both $0.4 \mathrm{~mA}$ (Fig. $5 A)\left(t_{(17)}=2.360 ; p=\right.$ $0.031)$ and $1 \mathrm{~mA}$ (Fig. $5 B)\left(t_{(10)}=2.243 ; p=0.049\right)$. As represented in Figure $5 E$, dorsal hippocampus endo- $\mathrm{N}$ injections effectively eliminated PSA-NCAM immunoreactivity on the fourth day after injection in an area around the infusion site covering the rostrocaudal extension of the hippocampus, from the very rostral 
A
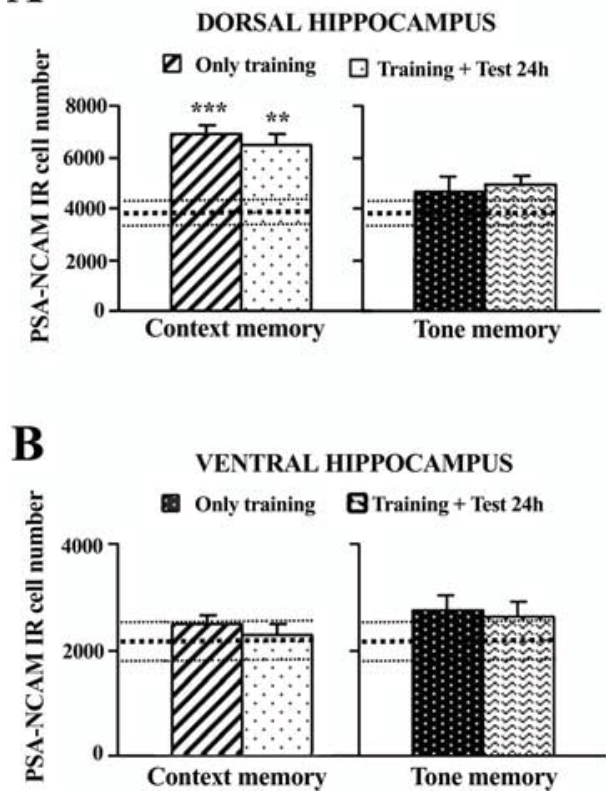

Figure 4. Upregulation of PSA-NCAM-labeled cells in the hippocampus induced by acquisition of contextual fear conditioning is specifically circumscribed to the dorsal, but not ventral, subdivision of the dentate gyrus. $\boldsymbol{A}$, Number of PSA-NCAMimmunoreactive cells in the granular cell layer of the dorsal dentate gyrus $24 \mathrm{~h}$ after training $(1 \mathrm{~mA})$ rats in a contextual (left) or in a tone (right) fear-conditioning paradigm. B, Number of PSA-NCAM-IR cells in the ventral subdivision of the dentate gyrus $24 \mathrm{~h}$ after contextual (right) or tone fear-conditioning (right) training (1 mA). C, Coronal brain sections illustrating the dorsal (left) and ventral (right) subdivisions of the hippocampal DG (red color) used to evaluate the frequency of cells expressing PSA-NCAM in these areas. Numbers indicate distance from bregma in millimeters. Data represent mean \pm SEM. $\boldsymbol{A}, \boldsymbol{B}$, Thickest horizontal discontinuous line represents mean values of the naive group and thinner ones represent SEM values of this group. ${ }^{* *} p<0.01$ and ${ }^{* * *} p<0.001$ versus naive.

edge of the hippocampus until $\sim 4-5 \mathrm{~mm}$ caudally. PSA removal was confined to the dorsal portion of the HF without affecting the ventral edge. Diffusion of the enzyme on the cortex just above the dorsal hippocampus was constantly found in the area around cannula placement, a feature reported for endo- $\mathrm{N}$ injections (Becker et al., 1996). Semiquantitative estimation of the extension of endo-N diffusion indicated that PSA cleavage in the dorsal hippocampus always included the whole DG and CA1 regions and $\sim 73 \%$ of the CA3 region. Figure $5 F$ shows PSA-NCAM immunoreactivity in a representative endo- $\mathrm{N}$-treated animal, and Figure $5 G$ shows a vehicle-injected control.

On the contrary, infusions of endo- $\mathrm{N}$ into the ventral hippocampus did not affect behavior in any of the fear-conditioning modalities ( $1 \mathrm{~mA}$ shock), as indicated by similar freezing levels in both vehicle-injected and endo-N-injected rats at both training and testing in both contextual [training, vehicle $=74.3 \pm 5.0$; endo- $\mathrm{N}=74.0 \pm 8.6$; retention test, vehicle $=79.8 \pm 2.5$; endo$\mathrm{N}=80.0 \pm 3.3 ; \mathrm{n} . \mathrm{s}$ ] and tone [training, vehicle $=84.2 \pm 2.8$; endo- $\mathrm{N}=84.7 \pm 3.1$; retention test, vehicle $=87.3 \pm 1.9$; endo$\mathrm{N}=83.4 \pm 1.6$; n.s] fear conditioning.

We then tested for the specificity of endo-N effects on contextual fear conditioning ( $1 \mathrm{~mA}$ ) by heat inactivating the enzyme and subsequently administering it into the dorsal hippocampus. Freezing levels of animals infused with inactivated endo- $\mathrm{N}(n=$ 9 ) were equivalent to those of vehicle-infused rats $(n=6)$, both at training (vehicle $=68.6 \pm 3.6$; inactivated endo- $\mathrm{N}=64.6 \pm 3.8$; n.s.) and at the $48 \mathrm{~h}$ retention test (vehicle $=65.2 \pm 2.6$; inactivated endo- $\mathrm{N}=64.3 \pm 2.8$; n.s.). After the experiment, all animals were perfused, and PSA-NCAM expression levels in the rostrocaudal extension of the DG were analyzed. No differences were found between vehicle and endo- $\mathrm{N}$-infused rats in the ex- pression levels of PSA-NCAM-IR cells, neither in dorsal nor in ventral or total hippocampus (data not shown).

Moreover, cleavage of hippocampal PSA-NCAM by posttraining injections of endo-N given immediately after CFC failed to affect subsequent freezing responses to context [retention test, vehicle $=74.6 \pm 9.3$; endo- $\mathrm{N}=77.7 \pm 7.3$; n.s].

\section{Enzymatic removal of PSA-NCAM in the dorsal hippocampus does not affect locomotion or unconditioned emotional behavior}

Finally, given that endo- $\mathrm{N}$ injections into the dorsal hippocampus reduced conditioned fear responses in the contextual paradigm, we designed an experiment to test whether they could affect nonspecific behavioral responses. Endo-N-injected animals showed locomotion and unconditioned fear/anxiety levels similar to those of vehicle-treated rats (Fig. 6), as indicated by their similar (1) percentage time spent in the center $\left(t_{(16)}=1.071\right.$; n.s.) (Fig. $6 \mathrm{~A}$, left) and total distance traveled $\left(t_{(16)}=\right.$ 0.313 ; n.s.) (Fig. $6 B$, right) in the open field, evaluated $48 \mathrm{~h}$ after injection; and (2) amplitude of startle responses to acoustic stimuli, evaluated $72 \mathrm{~h}$ after injection (session $1, t_{(16)}=0.902$, n.s.; session $2, t_{(16)}=$ 0.132 , n.s.) (Fig. $6 B$ ). Other behaviors scored in the open field, such as rearing, grooming, and freezing, as well as number of defecations, were also not altered by endo- $\mathrm{N}$ treatment (data not shown).

\section{Discussion}

Here, we present evidence for a specific involvement of PSANCAM in the dorsal hippocampus in CFC. We report an upregulation of PSA-NCAM expression in the dorsal (but not ventral) DG $24 \mathrm{~h}$ (but not $30 \mathrm{~min}$ ) after contextual (but not tone) fear conditioning. Microinfusion of the enzyme endo- $\mathrm{N}$ resulting in specific removal of PSA in the dorsal (but not ventral) hippocampus induced a marked reduction on freezing responses to the conditioned context. Although several of the PSA-labeled neurons observed at the granule cell layer of the adult DG are recently generated (Seki and Arai, 1993), the reported increase in PSANCAM-IR cells after CFC is mainly attributable to increased PSA expression in preexisting granule neurons, and not to neurogenesis, in agreement with Pham et al. (2005).

By combining behavior, immunohistochemistry, and a paradigm for PSA loss of function, we present several lines of evidence linking the specific upregulation of PSA-NCAM-IR cells observed in the dorsal DG with the mechanisms involved in the consolidation of the contextual fear memory: (1) The magnitude of PSA-NCAM increase in dorsal hippocampus was higher under the experimental conditions leading to the strongest memory. (2) The increase was observed at $24 \mathrm{~h}$, but not $30 \mathrm{~min}$, after training, in agreement with previous evidence showing DG PSA-NCAM upregulation occurring between 10 and $24 \mathrm{~h}$ after training rats in hippocampus-dependent tasks (Doyle et al., 1992; Murphy et al., 1996; Sandi et al., 2003; Venero et al., 2006), but not earlier (Mur- 
phy et al., 1996; Foley et al., 2003a,b). This timing is coherent with the role of PSANCAM in activity-induced synaptogenesis (Theodosis et al., 1999; Dityatev et al., 2004) and the time dynamics of synaptic changes that transiently occur in the DG during late stages of consolidation in other shock-related contextual tasks (O'Connell et al., 1997; O'Malley et al., 1998). (3) The increase was directly related to training and was not a mere activation induced by testing animals for retrieval. (4) The possibility that this effect was caused by stressrelated nonspecific effects, such as shock exposure, was discarded, because rats submitted to the immediate shock or to TFC failed to show changes in PSA-NCAM. (5) On the contrary, exposing animals just to the context (context exposure condition) induced an activation of PSA-NCAM in the dorsal DG (but also in the ventral DG; see discussion below), which supports a role of this neuroplastic mechanism in the DG in context representation. (6) Notably, enzymatic removal of PSA in the dorsal hippocampus by the highly PSA-specific glycosidase endo-N specifically inhibited contextual fear memory. Although injections were given $24 \mathrm{~h}$ pretraining [note that PSA cleavage takes $\sim 1 \mathrm{~d}$ to occur and remains downregulated for up to 1-4 weeks (data not shown) (Kiss et al., 1994; Becker et al., 1996; Seki and Rutishauser, 1998; Theodosis et al., 1999)], postshock freezing levels at training were not affected by endo-N infusions. Moreover, cleavage of PSA-NCAM by endo-N injections given after training did not affect subsequent retrieval, which again supports the implication of PSA-NCAM in mechanisms linked to memory consolidation and discards an effect of PSA cleavage purely on retrieval. Specificity of the effect of dorsal endo-N injections in contextual fear memory mechanisms is also supported by our results showing that the same treatment affected neither conditioned fear responses to the tone, nor locomotion, nor unconditioned emotional responses. Furthermore, specificity is confirmed by an additional experiment showing that the infusion of heat-inactivated endo- $\mathrm{N}$ into the dorsal hippocampus failed to mimic the impairing effects of endo- $\mathrm{N}$ in contextual fear conditioning, in parallel with its lack of effectiveness cleaving PSA.

Anatomically, the dorsal hippocampus is strategically placed to form a context representation, because it receives a major input of visuospatial information from association cortices and perirhinal and entorhinal areas (Moser and Moser, 1998). Our findings indicating a specific functional implication of PSANCAM in the dorsal hippocampus in contextual conditioning are in agreement with lesion studies that critically implicate the dorsal hippocampus in the mnemonic processes of CFC (see Introduction) and highlight PSA-NCAM among the mediating mechanisms. This role for PSA-NCAM is supported by evidence showing that enzymatic removal of hippocampal PSA impaired
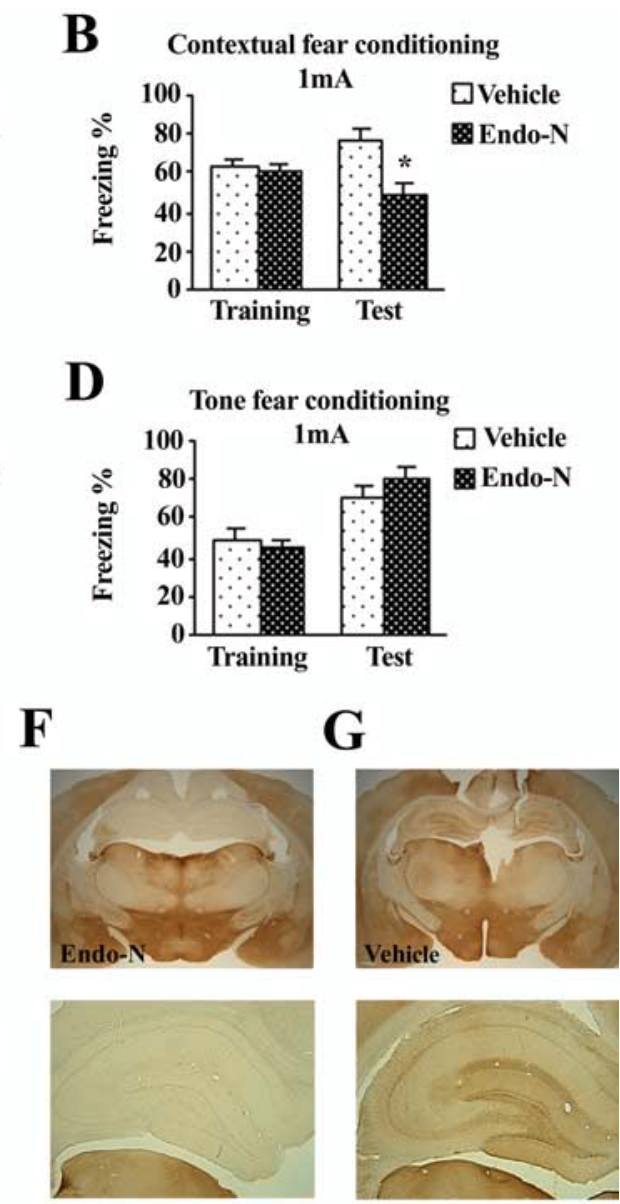

Figure 5. Enzymatic removal of PSA-NCAM in the dorsal hippocampus disrupts long-term establishment of contextual fear template show the representative diffusion of the enzyme across animals. Injection of endo- $N$ effectively eliminated PSA-NCAM around the infusion site, covering the rostrocaudal extension of the hippocampus from the very rostral edge until 4-5 mm caudally. Diffusion of the enzyme throughout the hippocampus covered the whole dorsal dentate gyrus and CA1 regions and Representative photomicrographs of a coronal section (top) and detail of the dentate gyrus (bottom) in an animal injected with endo-N or with vehicle, respectively. ${ }^{*} p<0.05$ versus vehicle-injected group. Data represent mean \pm SEM.

activity-dependent synaptic plasticity (Becker et al., 1996; Muller et al., 1996; Eckhardt et al., 2000) and associated structural synaptic remodeling (Dityatev et al., 2004), as well as spatial memory (Becker et al., 1996; Venero et al., 2006). It is also supported by studies showing that training-induced PSA upregulation in the DG is not observed in pseudotrained animals or in those rendered amnesic with scopolamine (Murphy et al., 1996). PSA acts as a steric regulator of cell-cell surface apposition, reducing cell adhesion and allowing plasticity (Rutishauser and Landmesser, 1996). Because memory consolidation is believed to require changes in gene transcription that are generally accompanied by synaptic remodeling (Martin et al., 2000), the role of PSA-NCAM in learning is in most cases attributable to its permissive action in synaptic plasticity (including its roles in neuritogenesis, synaptogenesis, and neurogenesis) [for reviews, see Kiss and Muller (2001) and Bonfanti (2006)]. Moreover, PSA-NCAM might also act by affecting signaling pathways involved in contextual learning. For example, recent evidence indicates that PSA-NCAM can 


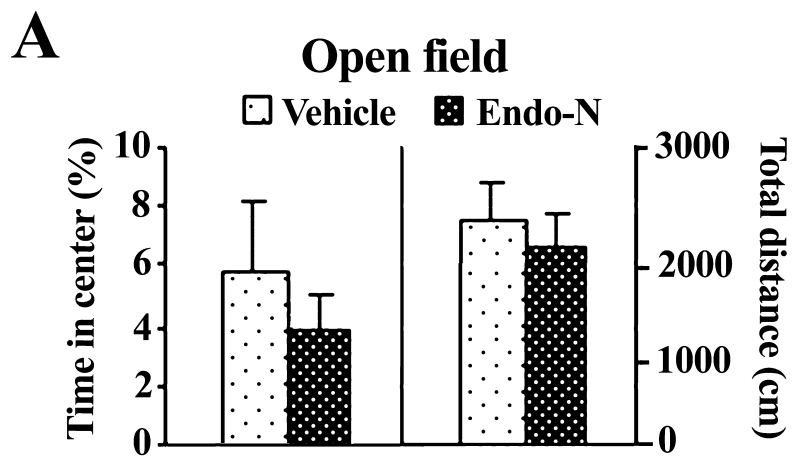

B

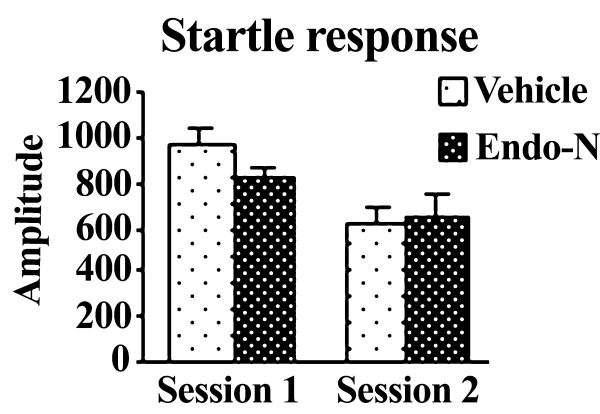

Figure 6. Intact locomotion, emotional reactivity, and startle response after bilateral injection of endo- $\mathrm{N}$ in the dorsal hippocampus. $\boldsymbol{A}$, Mean percentage \pm SEM of time rats spent exploring center $(\boldsymbol{A}$, left) and distance traveled ( $\boldsymbol{B}$, right) in the open field $48 \mathrm{~h}$ after enzyme injection. $\boldsymbol{B}$, Mean amplitude $\pm \mathrm{SEM}$ of startle response in two following sessions consisting of 10 randomly startle trials ( $115 \mathrm{~dB} ; 40 \mathrm{~ms}) 72 \mathrm{~h}$ after endo- $\mathrm{N}$ administration.

interact with different molecules that play a role in learning, such as NMDA receptors (Hammond et al., 2006), AMPA receptors (Vaithianathan et al., 2004), and brain-derived neurotrophic factor (Vutskits et al., 2001).

Because NMDA receptors in the dorsal hippocampus have been involved in the acquisition of contextual fear conditioning (Young et al., 1994; Bast et al., 2003), a question that arises from our study is whether the observed upregulation of PSA-NCAM after contextual fear conditioning is dependent or independent of NMDA receptor activation. Available evidence about the ability of NMDA receptors to modulate PSA-NCAM expression does not present a clear pattern, with some studies indicating a positive (Bouzioukh et al., 2001a,b), whereas others indicate a negative (Bouzioukh et al., 2001b; Nacher et al., 2002), regulation of PSA expression by NMDA. Future studies will be performed to directly address this question (i.e., whether context-induced upregulation of PSA-NCAM depends on NMDA receptor activation or whether it occurs in parallel involving different pathways).

As for the ventral hippocampus, our results bring new information to the debate about the role of this hippocampal subdivision in CFC. One view questions the contribution the ventral hippocampus makes to memory for contextual fear based on the fact that ventral lesions affect unconditioned anxiety (Gray and McNaughton, 2000; Bannerman et al., 2004), including unconditioned fear responses (Kjelstrup et al., 2002). Because this subdivision displays close interconnections with the amygdala (Moser and Moser, 1998), it has been considered a gateway that conveys contextual information between the dorsal hippocampus and the amygdala (Maren and Holt, 2004). An alternative view proposes that memory for a novel context is distributed throughout the longitudinal extent of the hippocampus, includ- ing the ventral part, and that this representation helps to support contextual fear memories (Rudy and Matus-Amat, 2005). Interestingly, some of the critical evidence in support of each view has been obtained with different CFC paradigms [i.e., the former with the standard CFC (association of context and shock in the same session) and the latter with the so-called CPFE]. In CPFE, contextual fear memories are formed by combining context exposure with immediate shock given $24 \mathrm{~h}$ afterward. By itself, immediate shock does not lead to any contextual fear memory, whereas context exposure induces a context memory that stays at least for 24 h (Fanselow, 2000; Rudy and O'Reilly, 2001; Rudy et al., 2002; Rudy and Wright-Hardesty, 2005). The fact that the standard CFC protocol leads to PSA-NCAM activation in the dorsal DG, whereas context exposure leads in addition to its activation in the ventral part, reinforces the view that CFC induced by the standard and the CPFE protocols involve different neurobiological mechanisms (Huff et al., 2005; Biedenkapp and Rudy, 2007).

Given the plasticity-promoting properties of PSA-NCAM (Bonfanti, 2006), it is tempting to speculate that the nature of operations performed by the ventral hippocampus during context exposure differ from those involved in the standard CFC protocol. Context exposure in the CPFE protocol leads to a stronger and more resistant representation of context (Biedenkapp and Rudy, 2007). Accordingly, our results indicate that context representation in the $\mathrm{CPFE}$ might involve supplementary plasticity mechanisms in the ventral hippocampus. They are congruent with reports indicating that contextual memories are disrupted by inactivation of the ventral hippocampus in the CPFE paradigm (Rudy and Matus-Amat, 2005) but frequently not in the standard CFC task (Kjelstrup et al., 2002). Importantly, the lack of involvement of PSA-NCAM in the ventral hippocampus in the standard CFC does not imply a lack of involvement of this hippocampal subdivision in this task; it only indicates that ventral hippocampal PSA-NCAM seems not to be required for this type of memory. This would fit with the general nonassociative operations proposed to occur in the ventral hippocampus during defensive behavior (Gray and McNaughton, 2000). Moreover, given the connection of the ventral hippocampus with the amygdala, it is noteworthy that amygdaloid PSA-NCAM seems also not to play a functional role in fear conditioning (Senkov et al., 2006; Markram et al., 2007a,b).

Except for a few reports suggesting a potential involvement of the hippocampus in TFC (Maren, 1999; Bast et al., 2001; Maren and Holt, 2004), other studies suggested that it is not critically involved in this type of conditioning (Bast et al., 2003; Sanders et al., 2003). Although TFC-trained animals also express conditioned fear of the context, this is both quantitatively and qualitatively different from that displayed by animals trained in the CFC version. In TFC, subjects process the discrete CS (tone) as the main predictor, attributing less predictive contextual cues to the "background." In contrast, in CFC, contextual cues are processed as valid predictors (context is in the "foreground"). The neural mechanisms mediating hippocampal involvement in fear conditioning seem to differ depending on whether the context is in the background or in the foreground (Calandreau et al., 2006; Trifilieff et al., 2006). Lesions to the hippocampus interfere with background but not foreground CFC (Phillips and LeDoux, 1994). Therefore, it is not surprising that the neuroplastic event under study here, namely PSA-NCAM, was not modulated by TFC and not required for the formation of TFC memories.

In summary, these data indicate that CFC promotes a specific upregulation of PSA-NCAM in the dorsal hippocampus, which is 
critically involved in plasticity processes occurring during consolidation of context fear memories.

\section{References}

Anagnostaras SG, Gale GD, Fanselow MS (2001) Hippocampus and contextual fear conditioning: recent controversies and advances. Hippocampus $11: 8-17$.

Angata K, Long JM, Bukalo O, Lee W, Dityatev A, Wynshaw-Boris A, Schachner M, Fukuda M, Marth JD (2004) Sialyltransferase ST8Sia-II assembles a subset of polysialic acid that directs hippocampal axonal targeting and promotes fear behaviour. J Biol Chem 279:32603-32613.

Bannerman DM, Rawlins JNP, McHugh SB, Deacon RMJ, Yee BK, Bast T, Zhang W-N, Pothuizen HHJ, Feldon J (2004) Regional dissociations within the hippocampus-memory and anxiety. Neurosci Biobehav Rev 28:273-283.

Bast T, Zhang W-N, Feldon J (2001) The ventral hippocampus and fear conditioning in rats: different anterograde amnesias of fear after tetrodotoxin inactivation and infusion of the $\mathrm{GABA}_{\mathrm{A}}$ agonist muscimol. Exp Brain Res 139:39-52.

Bast T, Zhang W-N, Feldon J (2003) Dorsal hippocampus and classical fear conditioning to tone and context in rats: effects of local NMDA-receptor blockade and stimulation. Hippocampus 13:657-675.

Becker CG, Artola A, Gerardy-Schahn R, Becker T, Welzl H, Schachner M (1996) The polysialic acid modification of the neural cell adhesion molecule is involved in spatial learning and hippocampal long-term potentiation. J Neurosci Res 45:143-152.

Biedenkapp JC, Rudy JW (2007) Context preexposure prevents forgetting of a contextual fear memory: implication for regional changes in brain activation patterns associated with recent and remote memory tests. Learn Mem 14:200-203.

Bonfanti L (2006) PSA-NCAM in mammalian structural plasticity and neurogenesis. Prog Neurobiol 80:129-164.

Bouzioukh F, Tell F, Jean A, Rougon G (2001a) NMDA receptor and nitric oxide synthase activation regulate polysialylated neural cell adhesion molecule expression in adult brainstem synapses. J Neurosci 21:4721-4730.

Bouzioukh F, Tell F, Rougon G, Jean A (2001b) Dual effects of NMDA receptor activation on polysialylated neural cell adhesion molecule expression during brainstem postnatal development. Eur J Neurosci 14:1194-1202.

Burgess A, Aubert I (2006) Polysialic acid limits choline acetyltransferase activity induced by brain-derived neurotrophic factor. J Neurochem 99:797-806.

Calandreau L, Trifilieff P, Mons N, Costes L, Marien M, Marighetto A, Micheau J, Jaffard R, Desmedt A (2006) Extracellular hippocampal acetylcholine level controls amygdala function and promotes adaptive conditioned emotional response. J Neurosci 26:13556-13566.

Cordero MI, Merino JJ, Sandi C (1998) Correlational relationship between shock intensity and corticosterone secretion on the establishment and subsequent expression of contextual fear conditioning. Behav Neurosci 112:885-891.

Cordero MI, Kruyt ND, Merino JJ, Sandi C (2002) Glucocorticoid involvement in memory formation in a rat model for traumatic memory. Stress 5:73-79.

Daston MM, Bastmeyer M, Rutishauser U, O’Leary DDM (1996) Spatially restricted increase in polysialic acid enhances corticospinal axon branching related to target recognition and innervation. J Neurosci 16:5488-5497.

Desmedt A, Marighetto A, Garcia R, Jaffard R (2003) The effects of ibotenic hippocampal lesions on discriminative fear conditioning to context in mice: impairment or facilitation depending on the associative value of a phasic explicit cue. Eur J Neurosci 17:1953-1963.

Dityatev A, Schachner M (2003) Extracellular matrix molecules and synaptic plasticity. Nat Rev Neurosci 4:456-468.

Dityatev A, Dityateva G, Sytnyk V, Delling M, Toni N, Nikonenko I, Muller D, Schachner M (2004) Polysialylated neural cell adhesion molecule promotes remodeling and formation of hippocampal synapses. J Neurosci 24:9372-9382.

Doyle E, Nolan PM, Bell R, Regan CM (1992) Hippocampal NCAM180 transiently increases sialylation during the acquisition and consolidation of a passive avoidance response in the adult rat. J Neurosci Res 31:513-523.

Eckhardt M, Bukalo O, Chazal G, Wang L, Goridis C, Schachner M, Gerardy-
Schahn R, Cremer H, Dityatev A (2000) Mice deficient in the polysialyltransferase ST8SiaIV/PST-1 allow discrimination of the roles of neural cell adhesion molecule protein and polysialic acid in neural development and synaptic plasticity. J Neurosci 20:5234-5244.

Fanselow MS (2000) Contextual fear, gestalt memories, and the hippocampus. Behav Brain Res 110:73-81.

Foley AG, Hedigan K, Roullet P, Moricard Y, Murphy KJ, Sara SJ, Regan CM (2003a) Consolidation of memory for odour-reward association requires transient polysialylation of the neural cell adhesion molecule in the rat hippocampal dentate gyrus. J Neurosci Res 74:570-576.

Foley AG, Ronn LC, Murphy KJ, Regan CM (2003b) Distribution of polysialylated neural cell adhesion molecule in rat septal nuclei and septohippocampal pathway: transient increase of polysialylated interneurons in the subtriangular septal zone during memory consolidation. J Neurosci Res 74:807-817.

Gould E, Beylin A, Tanapat P, Reeves A, Shors TJ (1999) Learning enhances adult neurogenesis in the hippocampal formation. Nat Neurosci 2:260-265.

Gray JA, McNaughton N (2000) The neuropsychology of anxiety: an enquiry in to the functions of the septo-hippocampal systems, Ed 2. Oxford: Oxford UP.

Hammond MSL, Sims C, Parameshwaran K, Suppiramaniam V, Schachner M, Dityatev A (2006) Neural cell adhesion molecule-associated polysialic acid inhibits NR2B-containing $N$-methyl-D-aspartate receptors and prevents glutamate-induced cell death. J Biol Chem 281:34859-34869.

Huff NC, Wright-Hardesty KJ, Higgins EA, Matus-Amat P, Rudy JW (2005) Context pre-exposure obscures amygdala modulation of contextual-fear conditioning. Learn Mem 12:456-460.

Kim JJ, Fanselow MS (1992) Modality-specific retrograde amnesia of fear. Science 256:675-677.

Kiss JZ, Muller D (2001) Contribution of the neural cell adhesion molecule to neuronal and synaptic plasticity. Rev Neurosci 12:297-310.

Kiss JZ, Rougon G (1997) Cell biology of polysialic acid. Curr Opin Neurobiol 7:640-646.

Kiss JZ, Wang C, Olive S, Rougon G, Lang J, Baetens D, Harry D, Pralong WF (1994) Activity-dependent mobilization of the adhesion molecule polysialic NCAM to the cell surface of neurons and endocrine cells. EMBO J 13:5284-5292.

Kjelstrup KG, Tuvnes FA, Steffenach HA, Murison R, Moser EI, Moser MB (2002) Reduced fear expression after lesions of the ventral hippocampus. Proc Natl Acad Sci USA 99:10825-10830.

Lee I, Kesner RP (2004) Differential contributions of dorsal hippocampal subregions to memory acquisition and retrieval in contextual fearconditioning. Hippocampus 14:301-310.

Lemaire V, Lamarque S, LeMoal M, Piazza PV, Abrous DN (2006) Postnatal stimulation of the pups counteracts prenatal stress-induced deficits in hippocampal neurogenesis. Biol Psychiatry 59:786-792.

Lenka F, Rutishauser U, Prosser R, Huaming S, Glass JD (2002) Removal of polysialic acid from the SCN potentiates nonphotic circadian phase resetting. Physiol Behav 77:361-369.

Maren S (1999) Neurotoxic or electrolytic lesions of the ventral subiculum produce deficits in the acquisition and expression of pavlovian fear conditioning in rats. Behav Neurosci 113:283-290.

Maren S, Holt WG (2004) Hippocampus and pavlovian fear conditioning in rats: muscimol infusions into the ventral, but not dorsal, hippocampus impair the acquisition of conditional freezing to an auditory conditional stimulus. Behav Neurosci 118:97-110.

Maren S, Aharonov G, Fanselow MS (1997) Neurotoxic lesions of the dorsal hippocampus and pavlovian fear conditioning in rats. Behav Brain Res $88: 261-274$.

Markram K, Gerardy-Schahn R, Sandi C (2007a) Selective learning and memory impairments in mice deficient for polysialylated NCAM in adulthood. Neuroscience 144:788-796.

Markram K, Lopez-Fernandez MA, Abrous N, Sandi C (2007b) Amygdala upregulation of NCAM polysialylation induced by fear conditioning is not required for fear memory processes. Neurobiol Learn Mem 87:573-582.

Martin SJ, Grimwood PD, Morris RG (2000) Synaptic plasticity and memory: an evaluation of the hypothesis. Annu Rev Neurosci 23:649-711.

Moser MB, Moser EL (1998) Functional differentiation in the hippocampus. Hippocampus 8:608-619.

Muller D, Wang C, Skibo G, Toni N, Cremer H, Calaora V, Rougon G, Kiss JZ 
(1996) PSA-NCAM is required for activity-induced synaptic plasticity. Neuron 17:413-422.

Murphy KJ, Regan CM (1998) Contributions of cell adhesion molecules to altered synaptic weightings during memory consolidation. Neurobiol Learn Mem 70:73-81.

Murphy KJ, O'Connell AW, Regan CM (1996) Repetitive and transient increases in hippocampal neural cell adhesion molecule polysialylation state following multitrial spatial training. J Neurochem 67:1268-1274.

Nacher J, Alonso-Llosa G, Rosell D, McEwen B (2002) PSA-NCAM expression in the piriform cortex of the adult rat. Modulation by NMDA receptor antagonist administration. Brain Res 927:111-121.

O'Connell AW, Fox GB, Barry T, Murphy KJ, Fichera G, Foley AG, Kelly J, Regan CM (1997) Spatial learning activates neural cell adhesion molecule polysialylation in a corticohippocampal pathway within the medial temporal lobe. J Neurochem 68:2538-2546.

O'Malley A, O'Connell C, Regan CM (1998) Ultrastructural analysis reveals avoidance conditioning to induce a transient increase in hippocampal dentate spine density in the 6 hour post-training period of consolidation. Neuroscience 87:607-613.

O’Reilly RC, Rudy JW (2001) Conjunctive representations in learning and memory: principles of cortical and hippocampal function. Psychol Rev 108:311-345.

Paxinos G, Watson C (1986) The rat brain in stereotaxic coordinates. London: Academic.

Pham K, McEwen BS, LeDoux JE, Nader K (2005) Fear learning transiently impairs hippocampal cell proliferation. Neuroscience 130:17-24.

Phillips RG, LeDoux JE (1992) Differential contribution of amygdale and hippocampus to cued and contextual fear conditioning. Behav Neurosci 106:274-285.

Phillips RG, LeDoux JE (1994) Lesions of the dorsal hippocampal formation interfere with background but not foreground contextual fear conditioning. Learn Mem 1:34-44.

Richmond MA, Yee BK, Pouzet B, Veenman L, Rawlins JN, Feldon J, Bannerman DM (1999) Dissociating context and space within the hippocampus: effects of complete, dorsal, and ventral excitotoxic hippocampal lesions on conditioned freezing and spatial learning. Behav Neurosci 113:1189-1203.

Rougon G, Dubois C, Buckley N, Magnani JL, Zollinger W (1986) A monoclonal antibody against meningococcus group B polysaccharides distinguishes embryonic from adult N-CAM. J Cell Biol 103:2429-2437.

Rudy JW, Matus-Amat P (2005) The ventral hippocampus supports a memory representation of context and contextual fear conditioning: implications for a unitary function of the hippocampus. Behav Neurosci 119:154-163.

Rudy JW, O’Reilly RC (2001) Conjunctive representations, the hippocampus, and contextual fear conditioning. Cogn Affect Behav Neurosci $1: 66-82$.

Rudy JW, Wright-Hardesty K (2005) The temporal dynamics of retention of a context memory: something is missing. Learn Mem 12:172-177.

Rudy JW, Barrientos RM, O’Reilly RC (2002) Hippocampal formation supports conditioning to memory of a context. Behav Neurosci 116:530-538.

Rudy JW, Huff NC, Matus-Amat P (2004) Understanding contextual fear conditioning insights from a two-process model. Neurosci Biobehav Rev 28:675-685.

Rutishauser U, Landmesser L (1996) Polysialic acid in the vertebrate ner- vous system: a promoter of plasticity in cell-cell interactions. Trends Neurosci 19:422-427.

Rutishauser U, Acheson A, Hall AK, Mann DM, Sunshine J (1988) The neural cell adhesion molecule (NCAM) as a regulator of cell-cell interactions. Science 240:53-57.

Sanders MJ, Wiltgen BJ, Fanselow MS (2003) The place of the hippocampus in fear conditioning. Eur J Pharmacol 463:217-223.

Sandi C, Merino JJ, Cordero MI, Kruyt ND, Murphy KJ, Regan CM (2003) Modulation of hippocampal NCAM polysialylation and spatial memory consolidation by fear conditioning. Biol Psychiatry 54:599-607.

Seki T, Arai Y (1993) Distribution and possible roles of the highly polysialylated neural cell adhesion molecule (NCAM-H) in the developing and adult central nervous system. Neurosci Res 17:265-290.

Seki T, Rutishauser U (1998) Removal of polysialic acid-neural cell adhesion molecule induces aberrant mossy fiber innervation and ectopic synaptogenesis in the hippocampus. J Neurosci 18:3757-3766.

Selden NRW, Everitt BJ, Jarrard LE, Robbins TW (1991) Complementary roles for the amygdale and hippocampus in aversive conditioning to explicit and contextual cues. Neuroscience 42:335-350.

Senkov O, Sun M, Weinhold B, Gerardy-Schahn R, Schachner M, Dityatev A (2006) Polysialylated neural cell adhesion molecule is involved in induction of long-term potentiation and memory acquisition and consolidation in a fear-conditioning paradigm. J Neurosci 26:10888-10989.

Sutherland RJ, Rudy JW (1989) Configural association theory: the role of the hippocampal formation in learning, memory, and amnesia. Psychobiology 17:129-144.

Theodosis DT, Bonhomme R, Vitiello S, Rougon G, Poulain DA (1999) Cell surface expression of polysialic acid on NCAM is a prerequisite for activity-dependent morphological neuronal and glial plasticity. J Neurosci 19:10226-10236.

Trifilieff P, Herry C, Vanhoutte P, Caboche J, Desmedt A, Riedel G, Mons N, Micheau J (2006) Foreground contextual fear memory consolidation requires two independent phases of hippocampal ERK/CREB activation. Learn Mem 13:349-358.

Vaithianathan T, Matthias K, Bahr B, Schachner M, Suppiramaniam V, Dityatev A, Steinhauser C (2004) Neural cell adhesion moleculeassociated polysialic acid potentiates alpha-amino-3-hydroxy-5methylisoxazole-4-propionic acid receptor currents. J Biol Chem 279:47975-47984.

Venero C, Herrero AI, Touyarot K, Cambon K, Lopez-Fernandez MA, Berezin V, Bock E, Sandi C (2006) Hippocampal up-regulation of NCAM expression and polysialylation plays a key role on spatial memory. Eur J Neurosci 23:1585-1595.

Vutskits L, Djebbara-Hannas Z, Zhang H, Paccaud JP, Durbec P, Rougon G, Muller D, Kiss JZ (2001) PSA-NCAM modulates BDNF-dependent survival and differentiation of cortical neurons. Eur J Neurosci 13:1391-1402.

Weinhold B, Seidenfaden R, Rockle I, Muhlenhoff M, Schertzinger F, Conzelmann S, Marth JD, Gerardy-Schahn R, Hildebrandt H (2005) Genetic ablation of polysialic acid causes severe neurodevelopmental defects rescued by deletion of the neural cell adhesion molecule. J Biol Chem 280:42971-42977.

Young SL, Bohenek DL, Fanselow MS (1994) NMDA processes mediate anterograde amnesia of contextual fear conditioning induced by hippocampal damage: immunization against amnesia by context preexposure. Behav Neurosci 108:19-29. 